\title{
Epoetin alfa in der Onkologie - aktuelle Entwicklungen
}

\section{Krebsassoziierte Anämie - Ursachen und Symptome}

Anämie ist die häufigste hämatologische Funktionsstörung bei Krebspatienten. Je nach Tumortyp sind bei Diagnosestellung 20-60\% aller Krebspatienten anämisch, wie Prof. Dr. Hartmut Link, Kaiserslautern, im Juni auf dem Kongress der Multinational Association for Supportive Care in Cancer (MASCC) in Berlin erläuterte. Die Ursachen für eine Anämie sind vielfältig. Neben den bekannteren Faktoren (Tumorblutungen, Hämolyse, Vitamin-B12- und Folsäuremangel, Knochenmarkinfiltration) können auch Veränderungen des Immunstatus eine Anämie hervorrufen. So inhibieren von Monozyten, Makrophagen oder T-Lymphozyten sezernierte Zytokine die Proliferation und Differenzierung erythroider
Vorläuferzellen und hemmen die Produktion von Erythropoetin in der Niere. Im Verhältnis zum Grad der vorliegenden Anämie weisen Tumorpatienten daher häufig inadäquat niedrige Erythropoetinspiegel auf, so Link. Darüber hinaus kann auch die zytostatische Behandlung eine Anämie auslösen bzw. verstärken.

Eine Anämie äußert sich in vielen physischen und psychischen Symptomen (Abb. 1), die nicht nur die Lebensqualität der Patienten, sondern auch deren Funktionsstatus beeinflussen können, was oftmals zu mangelnder Therapietreue und Behandlungsverzögerungen führt. Das belastendste Anämiesymptom ist die Fatigue, unter der etwa $80 \%$ der Tumorpatienten leiden. Wie in Studien gezeigt, kann die FatigueSymptomatik durch ein Anheben des Hb-Wertes auf über $12 \mathrm{~g} / \mathrm{dl}$ signifikant verbessert wer-
Abb. 1. Symptome der Anämie
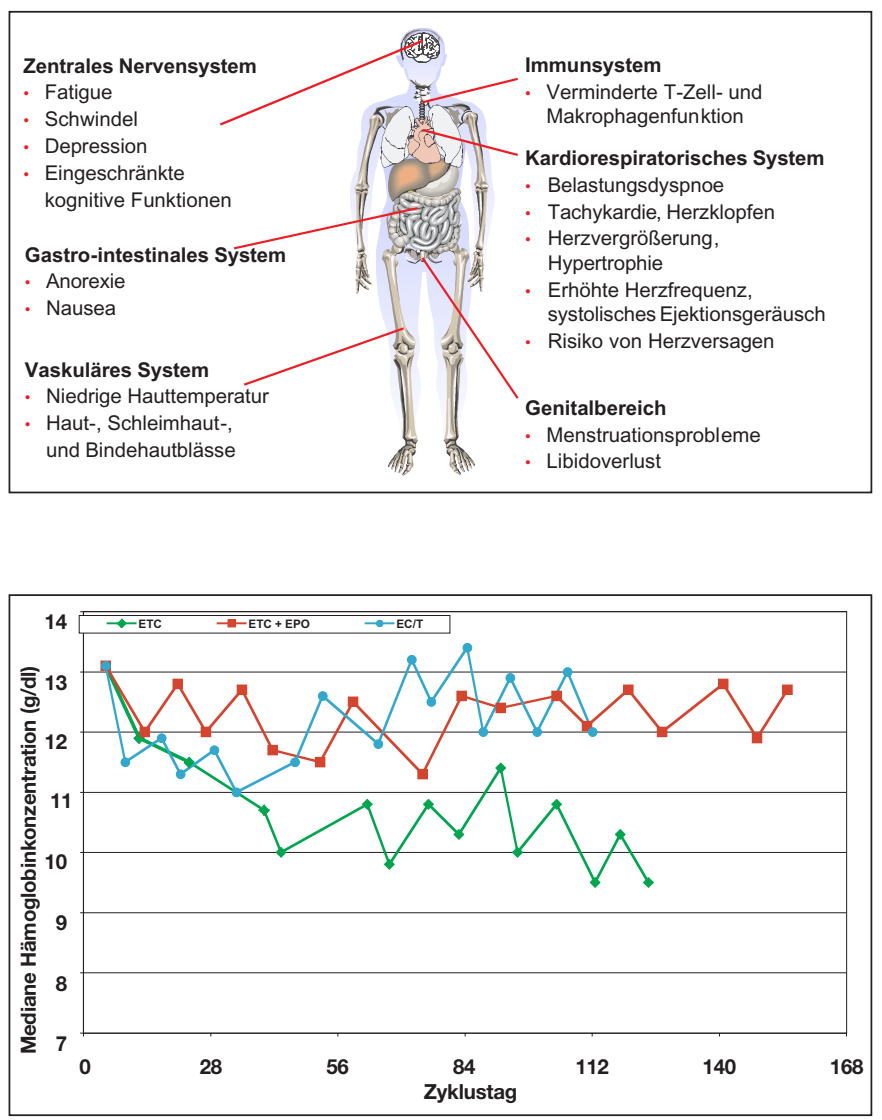

den. Dies kann durch die Gabe von Epoetin alfa selbst unter zytostatischer Behandlung erreicht werden.

\section{Evidenz-basierte Leitlinien zum Einsatz} von Epoetin

Für die Anämiekorrektur mit Epoetin in der Onkologie sind jetzt erstmals Evidenz-basierte Richtlinien der ASCO und ASH-Gremien verfügbar, die Prof. Dr. Mathias Freund, Rostock, beim MASCC vorstellte (s. Kasten).

Empfohlen wird Epoetin bei einer chemotherapieassoziierten Anämie mit einem $\mathrm{Hb}$-Wert $\leq 10$ $\mathrm{g} / \mathrm{dl}$. Dies gilt sowohl beim Vorliegen solider als auch hämatologischer Malignome. In Abhängigkeit vom Schweregrad der Anämie kann alternativ auch transfundiert werden. Zur Optimierung des Ansprechens sollte ein regelmäßiges Monitoring der Parameter Eisen, Eisenbindungskapazität, Transferrinsättigung und Ferritin erfolgen.

Zudem wird die Anwendung von Epoetin bei Patienten empfohlen, deren $\mathrm{Hb}$-Wert unter 12 $\mathrm{g} / \mathrm{dl}$ gefallen ist. Wie Freund erläuterte, beruht diese Einschätzung auf dem Einfluss des $\mathrm{Hb}$ Wertes auf die Lebensqualitätsparameter, die im Bereich zwischen 11 und $12 \mathrm{~g} / \mathrm{dl}$ die deutlichsten Verbesserungen zeigen.

Die Leitlinien empfehlen einen Ziel-Hb-Wert von $12 \mathrm{~g} / \mathrm{dl}$. Ist dieser Wert erreicht, sollte die Epoetin-Dosis reduziert werden. Die Anfangsdosierung von Epoetin liegt bei $3 \times 150 \mathrm{IE} / \mathrm{kg}$ $\mathrm{KG} /$ Woche für mindestens 4 Wochen. Bei Nichtansprechen kann eine Dosiserhöhung auf $3 \times 300 \mathrm{IE} / \mathrm{kg} \mathrm{KG} /$ Woche erfolgen. Da auch mit einer einmal wöchentlichen Dosis von $40000 \mathrm{IE}$ vergleichbar gute Ergebnisse erzielt wurden, kann auch diese Dosierung gewählt werden, so Freund.

Die Leitlinien basieren auf Literaturdaten der Jahre 1985-1999. Sie gelten daher nicht für die Verwendung von Darbepoetin alfa.

\section{Effektive Anämiekorrektur mit Epoetin alfa bei Tumorpatientinnen unter inten- sivierter Chemotherapie}

Zur Erhöhung der Therapieeffizienz in der Onkologie wurden in den vergangenen Jahren immer intensivere Behandlungsregimes eingeführt. Die verbesserte Wirksamkeit wird jedoch mit einer erhöhten Toxizität erkauft, die sich z.B. in höheren Anämieraten niederschlägt, was sich negativ auf Funktionsstatus und Prognose der Patienten auswirken kann. Dass eine Anämiekorrektur mit rekombinantem Epoetin alfa auch unter intensivierter zytostatischer Therapie effektiv ist, zeigen Daten, die im Rahmen des Senologie-Kongresses 2003 in München vorgestellt wurden

Prof. Dr. Volker Möbus, Frankfurt/Main, präsentierte Ergebnisse einer Phase-III-Studie mit 1284 Mammakarzinom-Patientinnen, in der $\mathrm{EC} \rightarrow \mathrm{T}$ (Epirubicin/Cyclophosphamid $\rightarrow$ Pacli- 
Abb. 3. Rezidivfreies Überleben (Epoetin alfa vs. Kontrollgruppe)

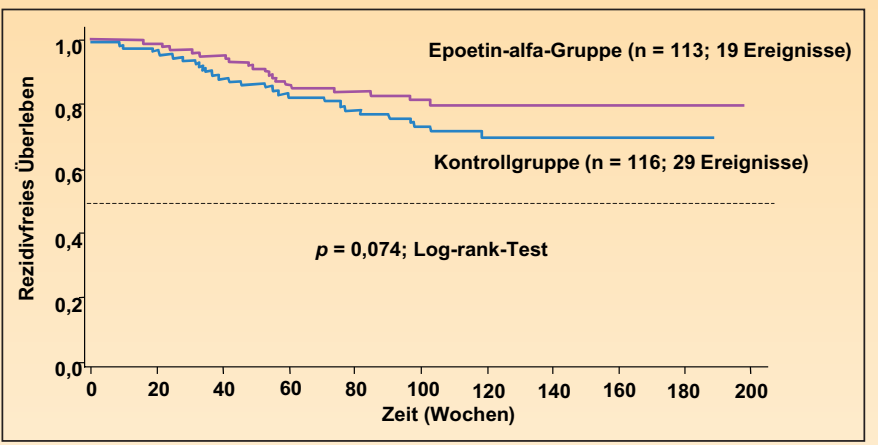

taxel, jeweils 90/600/175 mg/m², je 4 Zyklen) mit einem intervallverkürzten, dosisintensivierten ETC-Regime $\left(150 / 225 / 2500 \mathrm{mg} / \mathrm{m}^{2}\right.$, je 3 Zyklen) verglichen wurde. Im ETC-Arm erfolgte eine Subrandomisation $+/$ - Epoetin alfa $(3 \times 150 \mathrm{IE} / \mathrm{kg}$ KG/Woche). Während im konventionellen Arm fast keine Transfusionen benötigt wurden, waren bei $25 \%$ der Patientinnen im dosisintensivierten ETC-Arm ohne Epoetin alfa Transfusionen notwendig. Wurde zusätzlich zum ETC-

Regime Epoetin alfa gegeben, konnte dieser Anteil signifikant auf $11 \%$ gesenkt werden. $60 \%$ der mit Epoetin alfa behandelten Patientinnen, aber nur $23 \%$ der Patientinnen ohne Epoetin-alfa-Therapie wiesen einen Hb-Wert von $10-12 \mathrm{~g} / \mathrm{dl}$ auf. Der mediane Hb-Wert blieb mit Hilfe von Epoetin alfa auch während der ETC-Therapie mit 12,5 g/dl unverändert. Ohne Anämiekorrektur erfolgte dagegen unter ETC ein Abfall von 12,9 auf 10,6 g/dl (Abb. 2).

\section{ASCO / ASH-Leitlinien zum Einsatz von Epoetin in der Onkologie}

1. Epoetin wird für Patienten mit chemotherapieassoziierter Anämie ( $\mathrm{Hb} \leq 10 \mathrm{~g} / \mathrm{dl})$ empfohlen. In Abhängigkeit vom Schweregrad der Anämie oder den klinischen Umständen können als Alternative auch Transfusionen in Betracht gezogen werden.

Grad der Evidenz und der Empfehlung: IIB

2. Patienten, bei denen der Hb-Wert auf $<12 \mathrm{~g} / \mathrm{dl}$ gefallen ist, können sofort Epoetin erhalten, es muss nicht bis zum Abfall auf $\leq 10 \mathrm{~g} / \mathrm{dl}$ gewartet werden. Unter kritischen Bedingungen kann auch hier der Einsatz von Bluttransfusionen angezeigt sein. Grad der Evidenz und der Empfehlung: IIC

3. Die Empfehlungen zur Therapie der Anämie mit Epoetin basieren auf Studien, in denen $3 \times /$ Woche eine Dosis von 150 I.E./kg KG s.c. über einen Zeitraum von mindestens 4 Wochen verabreicht wurde. Alternativ dazu kann auch $1 \times /$ Woche eine Dosis von 40000 I.E. angewendet werden.

Grad der Evidenz und der Empfehlung: IIB

4. Bei Nichtansprechen auf Epoetin $(\Delta \mathrm{Hb}<1-2 \mathrm{~g} / \mathrm{dl}$ nach 6-8 Wochen und Dosiseskalation) sollte die Therapie abgebrochen werden. Zuvor ist jedoch abzuklären, ob eine Progression der Erkrankung oder ein Eisenmangel vorliegt.

Grad der Evidenz und der Empfehlung: Evidenzschlüssel nicht anwendbar (Evidenz basiert auf Expertenmeinung aufgrund indirekter Evidenz oder biologischer Interferenz), die Empfehlung ist Konsensus des Ausschusses.

5. Der Ziel-Hb-Wert beträgt $12 \mathrm{~g} / \mathrm{dl}$. Die Erhaltungsdosis sollte so titriert werden, dass dieser Wert gehalten wird.

Grad der Evidenz und der Empfehlung: Evidenzschlüssel nicht anwendbar (Evidenz basiert auf Expertenmeinung aufgrund indirekter Evidenz oder biologischer Interferenz), die Empfehlung ist Konsensus des Ausschusses.

6. Zur Optimierung des Ansprechens auf Epoetin sollten Eisen, Eisenbindungskapazität, Transferrinsättigung und Ferritin zu Beginn und im Verlauf der Behandlung kontrolliert werden. Gegebenenfalls ist Eisen zuzuführen.

Grad der Evidenz und der Empfehlung: Evidenzschlüssel nicht anwendbar (Evidenz basiert auf Expertenmeinung aufgrund indirekter Evidenz oder biologischer Interferenz), die Empfehlung ist Konsensus des Ausschusses.

7. Patienten mit Multiplem Myelom, Non-Hodgkin-Lymphomen und CLL, die eine Chemotherapie erhalten, sollten wie oben empfohlen mit Epoetin behandelt werden. Grad der Evidenz und der Empfehlung: IIB

8. Epoetin sollte bei Patienten mit Multiplem Myelom, Non-Hodgkin-Lymphomen und CLL gegeben werden, wenn der Hb-Wert infolge der Chemotherapie nicht ansteigt.

Grad der Evidenz und der Empfehlung: IVC
Verbessert Epoetin alfa die Prognose bei Zervixkarzinom-Patientinnen unter Radiochemotherapie?

Dass eine Anämiekorrektur mit Epoetin alfa auch bei Zervixkarzinom-Patientinnen unter sequenzieller adjuvanter Radiochemotherapie möglich ist, zeigte Prof. Dr. Jens-Uwe Blohmer, Berlin. In die gemeinsame Studie der AGO und NOGGO wurden 257 Hochrisikopatientinnen nach einer Wertheim-Operation aufgenommen. Sie erhielten sequenziell je 4 Zyklen Ifosfamid $\left(1,6 \mathrm{~m}^{2}\right.$, Tage 1-3) sowie Carboplatin (AUC 4, Tag 1) im Abstand von 3 Wochen, gefolgt von einer perkutanen Radiotherapie (50,4 Gy). Epoetin alfa wurde randomisiert in einer Dosis von $3 \times 10000 \mathrm{IE} /$ Woche appliziert.

Während in der Epoetin-alfa-Gruppe der mittlere $\mathrm{Hb}$-Wert unter der Chemotherapie von 12,0 auf $12,5 \mathrm{~g} / \mathrm{dl}$ anstieg, fiel er im Kontrollarm von 11,8 auf $10,8 \mathrm{~g} / \mathrm{dl}$. Im Epoetin-alfa-Arm verringerte sich hierdurch die Zahl der Grad-II-Anämien und der benötigten Transfusionen signifikant.

Noch interessanter war, dass die Lokalrezidivrate nach 90 Wochen im Epoetin-alfa-Arm nur $17 \%$ betrug, verglichen mit $25 \%$ im Kontrollarm (Abb. 3). Bestätigt sich dieser Trend, ist dies ein weiterer Beleg dafür, dass der Hb-Wert ein wesentlicher prognostischer Faktor bei der Radiotherapie des Zervixkarzinoms ist.

\section{Quelle}

Satelliten-Symposium «Hb 12: Erfolg in Therapie und Prognose mit Erypo ${ }^{\circledast}$ » 23. Jahrestagung der Deutschen Gesellschaft für Senologie, München, 19. Juni 2003.

und

Satelliten-Symposium «Evidence-Based Guidelines for Clinical Practice», Kongress der Multinational Association for Supportive Care in Cancer (MASCC), Berlin, 19. Juni 2003.

\section{Impressum \\ Erythropoetin in der Onkologie \\ - aktuelle Entwicklungen \\ PharmaForum in Onkologie 26 | 5 | 03}

(C) 2003 by S. Karger Verlag

Verlag für Medizin und Naturwissenschaften $\mathrm{GmbH}$

Lörracher Straße 16a

D-79115 Freiburg

Tel. +49761452070

E-mail Information@Karger.de

Mit freundlicher Unterstützung der Ortho Biotech, Division of Janssen-Cilag GmbH, Neuss

Der Verlag und die Herausgeber der Zeitschrift übernehmen keine Verantwortung für diese Rubrik.

Die Wiedergabe von Gebrauchsnamen, Handelsnamen, Warenbezeichnungen usw. in dieser Zeitschrift berechtigt auch ohne besondere Kennzeichnung nicht zur Annahme, dass solche Namen im Sinne der Warenzeichen- und Markenschutz-Gesetzgebung als frei zu betrachten wären und daher von jedermann benutzt werden dürfen. Für Angaben von Dosierungsanweisungen und Applikationsformen kann vom Verlag keine Haftung übernommen werden. Derartige Angaben müssen vom jeweiligen Anwender im Einzelfall anhand anderer Literaturstellen auf ihre Richtigkeit überprüft werden. 